\title{
Algebras, Derivations and Integrals
}

\author{
R. Casalbuoni币 \\ Département de Physique Théorique, Université de Genève \\ CH-1211 Genève 4, Suisse \\ e-mail: CASALBUONI@FI. INFN. IT
}

\begin{abstract}
In the context of the integration over algebras introduced in a previous paper, we obtain several results for a particular class of associative algebras with identity. The algebras of this class are called self-conjugated, and they include, for instance, the paragrassmann algebras of order $p$, the quaternionic algebra and the toroidal algebras. We study the relation between derivations and integration, proving a generalization of the standard result for the Riemann integral about the translational invariance of the measure and the vanishing of the integral of a total derivative (for convenient boundary conditions). We consider also the possibility, given the integration over an algebra, to define from it the integral over a subalgebra, in a way similar to the usual integration over manifolds. That is projecting out the submanifold in the integration measure. We prove that this is possible for paragrassmann algebras of order $p$, once we consider them as subalgebras of the algebra of the $(p+1) \times(p+1)$ matrices. We find also that the integration over the subalgebra coincides with the integral defined in the direct way. As a by-product we can define the integration over a one-dimensional Grassmann algebra as a trace over $2 \times 2$ matrices.
\end{abstract}

UGVA-DPT 1998/03-1000

PACS: 02.10, 02.10.S, 03.65.F

\footnotetext{
${ }^{1}$ On leave from Dipartimento di Fisica, Università di Firenze, I-50125 Firenze, Italia
} 


\section{Introduction}

Quantum mechanics has modified in a very profound way the classical understanding of the phase space of a physical system, making it non-commutative. This is reflected in a drastical change of the mathematics involved by promoting the classical phase space variables to operators acting on a Hilbert space. However, the flavor of the classical description is retained in the pathintegral formulation of quantum mechanics. In this case, although in the context of a rather different physical interpretation, one retains the concept of trajectories in the phase space. The situation changed again with the discovery of supersymmetric theories [1]. Although there are no experimental hints about their physical reality, the beauty of the mathematical structure involved has lead to an enormous amount of efforts in their understanding. But then, being supersymmetry tied to space-time invariance, one has to give up also to path-integration in terms of commuting variables. The space where the Feynman trajectories are defined gets enlarged to involve anticommuting variables (elements of a Grassmann algebra) related in an unavoidable way to the phase space coordinates by supersymmetry transformations. Also, considering the simple example of the supersymmetric particle [2], one realizes that due to the constraints involved, the space-time variables, $x_{\mu}$, loose their commutation properties. This aspect is not often emphasized, because it can be avoided by requiring the constraints to be satisfied as conditions on the physical states (the definition of chiral superfields), rather than solve them directly [2]. Finally the matrix realization of the $M$-theory introduces non-commuting coordinates (matrix-valued) for the D0-branes [3]. Following these considerations we found interesting to introduce, for a general algebra, the concept of integration [4], since it would play a vital role in the definition of the path-integration for these more general theories. To this end we started from the general approach to noncommutative geometry [5, 6]. That is from the observation that, in the commutative case, a space can be reconstructed from the algebra of its functions. Starting directly with the algebraic structure one can face situations where there are no concrete realizations of the space. Said that we have still to find a way to define the integration over an algebra. This can be done by lifting up to the algebra level the concept of integration over the space. To this end, let us look at the physics beyond the path-integral formalism. The physical amplitudes satisfy the composition law in an automatic way within this formalism, and this 
arises from the completeness relation which, in the case of a one-dimensional system, reads

$$
\int|x\rangle\langle x| d x=1
$$

Suppose that we have a set of orthonormal states in our Hilbert space, $\left\{\left|\psi_{n}\right\rangle\right\}$. Then we can convert the completeness relation in the space $\mathbb{R}^{1}$ into the orthogonality relation for the wave functions $\psi_{n}(x)=\left\langle x \mid \psi_{n}\right\rangle$,

$$
\int\left\langle\psi_{m} \mid x\right\rangle\left\langle x \mid \psi_{n}\right\rangle d x=\int \psi_{m}^{*}(x) \psi_{n}(x)=\delta_{m n}
$$

On the other side, given this equation, and the completeness relation for the set $\left\{\left|\psi_{n}\right\rangle\right\}$, we can reconstruct the completeness in the original space $\mathbb{R}^{1}$, that is the integration over the line. Now, we can translate the previous properties of the set $\left\{\left|\psi_{n}\right\rangle\right\}$, in the following two statements

1. The set of functions $\left\{\psi_{n}(x)\right\}$ span a vector space.

2. The product $\psi_{n}(x) \psi_{m}(x)$ can be expressed as a linear combination of the functions $\psi_{n}(x)$, since the set $\left\{\psi_{n}(x)\right\}$ is complete.

All this amounts to say that the set $\left\{\psi_{n}(x)\right\}$ is a basis of an algebra. In order to capture completely the context of eq. (1.2), we need also to understand the general meaning of $\psi_{n}^{*}(x)$. From the completeness it follows that $\psi_{n}^{*}(x)$ can be expressed as a linear combination of the functions $\psi_{n}(x)$,

$$
\psi_{n}^{*}(x)=\sum_{m} \psi_{m}(x) C_{m n}
$$

The matrix $C$ has to satisfy certain conditions that we will discuss in the text. In the following we will consider associative algebras with identity and with a matrix $C$ satisfying suitable conditions. These algebras will be called self-conjugated. In these cases we will define the integral over the algebra by eq. (1.2)

$$
\int_{(x)} \sum_{j} x_{j} C_{j i} x_{k}=\delta_{i k}
$$

where $\left\{x_{i}\right\}$ is a basis of the algebra. The properties that the $C$ matrix has to satisfy are such that the integral of an arbitrary element of the algebra, $\int_{(x)} x_{i}$, must be compatible with (1.4) and with the algebra product. This will be 
discussed in the following Section. This procedure and his motivations have been widely illustrated, in many examples, in ref. [4]. There we discussed also how to deal with important cases as the bosonic oscillator, or the $q$ bosonic oscillator algebras, where a suitable $C$ matrix does not exist. In this paper we will be interested in discussing some general result valid for self-conjugated algebras. In more detail we will prove the following results:

1. A theorem relating derivations on the algebra satisfying the integration by part rule (the vanishing of the integral of the derivation of an arbitrary element of the algebra) and automorphisms leaving invariant the integration measure. This is an extension of the theorem relating the invariance of the Riemann integral under translations and the vanishing of a total derivative (for convenient boundary conditions). We stress this point for its relevance within the path-integral approach, where the validity of the Schwinger's quantum principle depends precisely on the validity of this theorem.

2. All inner derivations, that is the derivations given by commutators, satisfy the integration by part rule.

3. The algebra of the $N \times N$ matrices, $\mathcal{A}_{N}$, is a self-conjugated algebra, with the integration given by the trace. The integration by part rule corresponds here to the cyclic property of the trace.

4. Given the integral over an algebra one can think of inducing it on a subalgebra. This is done in the particular case of a paragrassmann algebra of order $p, \mathcal{G}_{p}$, (that is generated by an element $\theta$ such that $\left.\theta^{p+1}=0\right)$ thought as a subalgebra of $\mathcal{A}_{p+1}$, the algebra of the $(p+1) \times$ $(p+1)$ matrices. The idea is to project out the subalgebra from the algebra, as one defines the integration over a submanifold by projecting it out from the manifold. In fact we will express the corresponding integral as a trace of the representative of the elements of $\mathcal{G}_{p}$ in $\mathcal{A}_{p+1}$, times an operator which projects out $\mathcal{G}_{p}$ from $\mathcal{A}_{p+1}$. In particular, this will allow us to get the integral over a Grassmann algebra $(p=1)$ as a trace over $2 \times 2$ matrices.

This paper is organized as follows: in Section 2 we will recall the main concepts necessary to define the integration over an algebra [4]. In Section 
3 we will study the relation between self-conjugated and involutive algebras. In Section 4 we will define the integration over the algebra of the $N \times N$ matrices. In Section 5 we will introduce the concept of derivation and we will derive the results 1), 2) and 3) mentioned above. The result 4) will be obtained in Section 6.

\section{Algebraic integration}

We recall here some of the concepts introduced in [4], in order to define the integration rules over a generic algebra. We start by considering an algebra $\mathcal{A}$ given by $n+1$ basis elements $x_{i}$, with $i=0,1, \cdots n$ (we do not exclude the possibility of $n \rightarrow \infty$, or of a continuous index). We assume the multiplication rules

$$
x_{i} x_{j}=f_{i j k} x_{k}
$$

with the usual convention of sum over the repeated indices. For the future manipulations it is convenient to organize the basis elements $x_{i}$ of the algebra in a ket

$$
|x\rangle=\left(\begin{array}{c}
x_{0} \\
x_{1} \\
\cdot \\
\cdot \\
x_{n}
\end{array}\right)
$$

or in the corresponding bra

$$
\langle x|=\left(\begin{array}{llll}
x_{0} & x_{1} & \cdots & x_{n}
\end{array}\right)
$$

Important tools for the study of a generic algebra are the right and left multiplication algebras. We define the associated matrices by

$$
R_{i}|x\rangle=|x\rangle x_{i}, \quad\langle x| L_{i}=x_{i}\langle x|
$$

For a generic element $a=\sum_{i} a_{i} x_{i}$ of the algebra we have $R_{a}=\sum_{i} a_{i} R_{i}$, and a similar equation for the left multiplication. In the following we will use also

$$
L_{i}^{T}|x\rangle=x_{i}|x\rangle
$$


The matrix elements of $R_{i}$ and $L_{i}$ are obtained from their definition

$$
\left(R_{i}\right)_{j k}=f_{j i k}, \quad\left(L_{i}\right)_{j k}=f_{i k j}
$$

The algebra is completely characterized by the structure constants. The matrices $R_{i}$ and $L_{i}$ are just a convenient way of encoding their properties. In the following we will be interested in associative algebras. By using the associativity condition

$$
x_{i}\left(x_{j} x_{k}\right)=\left(x_{i} x_{j}\right) x_{k}
$$

one can easily show the following relations (all equivalent to the previous relation)

$$
R_{i} R_{j}=f_{i j k} R_{k}, \quad L_{i} L_{j}=f_{i j k} L_{k}, \quad\left[R_{i}, L_{j}^{T}\right]=0
$$

The first two say that $R_{i}$ and $L_{i}$ are linear representations of the algebra, called the regular representations. The third that the right and left multiplications commute for associative algebras. In this paper we will be interested in associative algebras with identity, and such that there exists a matrix $C$, satisfying

$$
\begin{gathered}
L_{i}=C R_{i} C^{-1} \\
C^{T}=C
\end{gathered}
$$

We will call these algebras self-conjugated. The condition (2.9) is consistent with $L_{i}$ and $R_{i}$ satisfying the same algebra (see eq. (2.8)). Therefore, the non existence of the matrix $C$ boils down to the possibility that the algebra admits inequivalent regular representations. This happens, for instance, in the case of the bosonic algebra [4]. The condition of symmetry on $C$ can be interpreted in terms of the opposite algebra $\mathcal{A}^{D}$, defined by

$$
x_{i}^{D} x_{j}^{D}=f_{j i k} x_{k}^{D}
$$

The left and right multiplication in the dual algebra are related to those in $\mathcal{A}$ by

$$
R_{i}^{D}=L_{i}^{T}, \quad L_{i}^{D}=R_{i}^{T}
$$

Therefore the matrices $L_{i}^{T}$ are a representation of the dual algebra

$$
L_{i}^{T} L_{j}^{T}|x\rangle=x_{j} x_{i}|x\rangle=f_{j i k} L_{k}^{T}|x\rangle
$$


We see that the condition $C^{T}=C$ is equivalent to require that the relation (2.9) holds also for the right and left multiplication in the opposite algebra

$$
L_{i}^{D}=C R_{i}^{D} C^{-1}
$$

Since we are considering associative algebras, the requirement of existence of an identity is not a strong one, because we can always extend the given algebra to another associative algebra with identity. In fact, let us call $F$ the field over which the algebra is defined (usually $F$ is the field of real or complex numbers). Then, the extension of $\mathcal{A}$ (call it $\mathcal{A}_{1}$ ) is defined by the pairs

$$
(\alpha, a) \in \mathcal{A}_{1}, \quad \alpha \in F, \quad a \in \mathcal{A}
$$

with the product rule

$$
(\alpha, a)(\beta, b)=(\alpha \beta, \alpha a+\beta b+a b)
$$

The identity in $\mathcal{A}_{1}$ is given by the pair

$$
I=(1,0)
$$

Of course, this is the same as adding to any element of $\mathcal{A}$ a term proportional to the identity, that is

$$
\alpha I+a
$$

and defining the multiplication by distributivity. One can check easily that $\mathcal{A}_{1}$ is an associative algebra. An extension of this type exists also for many other algebras, but not for all. For instance, in the case of a Lie algebra one cannot add an identity with respect to the Lie product (since $I^{2}=0$ ). For self-conjugated algebras, $R_{i}$ has an eigenbra given by

$$
\langle x C|=\langle x| C, \quad\langle x C| R_{i}=x_{i}\langle x C|
$$

as it follows from (2.9) and (2.4). Then, as explained in the Introduction, we define the integration for a self-conjugated algebra by the formula

$$
\int_{(x)}|x\rangle\langle x C|=1
$$

where 1 is the identity in the space of the linear mappings on the algebra. In components the previous definition means

$$
\int_{(x)} x_{i} x_{k} C_{k j}=f_{i k p} C_{k j} \int_{(x)} x_{p}=\delta_{i j}
$$


This equation is meaningful only if it is possible to invert it in terms of $\int_{(x)} x_{p}$. This is indeed the case if $\mathcal{A}$ is an algebra with identity (say $x_{0}=I$ ) [回], because by taking $x_{i}=I$ in eq. (2.21), we get

$$
\int_{(x)} x_{j}=\left(C^{-1}\right)_{0 j}
$$

We see now the reason for requiring the condition (2.9). In fact it ensures that the value (2.22) of the integral of an element of the basis of the algebra gives the solution to the equation (2.21). In fact we have

$$
\int_{(x)} x_{i} x_{k} C_{k j}=f_{i k p} C_{k j} C_{0 p}^{-1}=\left(C^{-1} L_{i} C\right)_{0 j}=\left(R_{i}\right)_{0 j}=f_{0 i j}=\delta_{i j}
$$

as it follows from $x_{0} x_{i}=x_{i}$. Notice that the symmetry of $C$ allows us to write the integration as

$$
\int_{(x)}|x C\rangle\langle x|=1
$$

which is the form we would have obtained if we had started with the same assumptions but with the transposed version of eq. (2.4). All the examples considered in ref. [4], where the $C$ matrix exists, turn out to correspond to self-conjugated algebras. The examples we are referring to are the algebra over the circle, the paragrassmann algebras of order $p$, and the quaternionic algebra. In ref. [7] we have considered noncommuting toroidal algebras, which also turn out to be self-conjugated ones [8].

We will define an arbitrary function on the algebra by

$$
f(x)=\sum_{i} f_{i} x_{i} \equiv\langle x \mid f\rangle
$$

and its conjugated as

$$
f^{*}(x)=\sum_{i j} \bar{f}_{i} x_{j} C_{j i}=\langle f \mid x C\rangle
$$

where

$$
|f\rangle=\left(\begin{array}{c}
f_{0} \\
f_{1} \\
\cdot \\
\cdot \\
x_{n}
\end{array}\right), \quad\langle f|=\left(\begin{array}{llll}
\bar{f}_{0} & \bar{f}_{1} & \cdots & \bar{f}_{n}
\end{array}\right)
$$


and $\bar{f}_{i}$ is the complex-conjugated of the coefficient $f_{i}$ belonging to the field $\mathbb{C}$. Then a scalar product on the algebra is given by

$$
\langle f \mid g\rangle=\int_{(x)}\langle f \mid x C\rangle\langle x \mid g\rangle=\sum_{i} \bar{f}_{i} g_{i}
$$

\section{Algebras with involution}

In some case, as for the toroidal algebras [7], the matrix $C$ turns out to define a mapping which is an involution of the algebra. Let us consider the property of the involution on a given algebra $\mathcal{A}$. An involution is a linear mapping * $: \mathcal{A} \rightarrow \mathcal{A}$, such that

$$
\left(x^{*}\right)^{*}=x, \quad(x y)^{*}=y^{*} x^{*}, \quad x, y \in \mathcal{A}
$$

Furthermore, if the definition field of the algebra is $\mathbb{C}$, the involution acts as the complex-conjugation on the field itself. Given a basis $\left\{x_{i}\right\}$ of the algebra, the involution can be expressed in terms of a matrix $C$ such that

$$
x_{i}^{*}=x_{j} C_{j i}
$$

The eqs. (3.1) imply

$$
\left(x_{i}^{*}\right)^{*}=x_{j}^{*} C_{j i}^{*}=x_{k} C_{k j} C_{j i}^{*}
$$

from which

$$
C C^{*}=1
$$

From the product property applied to the equality

$$
R_{i}|x\rangle=|x\rangle x_{i}
$$

we get

$$
\left(R_{i}|x\rangle\right)^{*}=\left\langle x^{*}\right| R_{i}^{\dagger}=\langle x| C R_{i}^{\dagger}=\left(|x\rangle x_{i}\right)^{*}=x_{i}^{*}\left\langle x^{*}\right|=x_{i}^{*}\langle x| C
$$

and therefore

$$
\langle x| C R_{i}^{\dagger} C^{-1}=x_{j} C_{j i}\langle x|=\langle x| L_{j} C_{j i}
$$

that is

$$
C R_{i}^{\dagger} C^{-1}=L_{j} C_{j i}
$$


or also

$$
C R_{x_{i}}^{\dagger} C^{-1}=L_{x_{i}^{*}}
$$

If $R_{i}$ and $L_{i}$ are ${ }^{*}$-representations, that is

$$
R_{x_{i}}^{\dagger}=R_{x_{i}^{*}}=R_{x_{j}} C_{j i}
$$

we obtain

$$
C R_{x_{i}}^{\dagger} C^{-1}=C R_{x_{i}^{*}} C^{-1}=L_{x_{i}^{*}}
$$

Since the involution is non-singular, we get

$$
C R_{i} C^{-1}=L_{i}
$$

and comparing with the adjoint of eq. (3.11), we see that $C$ is a unitary matrix which, from eq. (3.4), implies $C^{T}=C$. Therefore we have the theorem:

Given an associative algebra with involution, if the right and left multiplications are ${ }^{*}$-representations, then the algebra is self-conjugated.

In this case our integration is a state in the Connes terminology [5].

If the $C$ matrix is an involution we can write the integration as

$$
\int_{(x)}|x\rangle\left\langle x^{*}\left|=\int_{(x)}\right| x^{*}\right\rangle\langle x|=1
$$

\section{The algebra of matrices}

Since an associative algebra admits always a matrix representation, it is interesting to consider the definition of the integral over the algebra $\mathcal{A}_{N}$ of the $N \times N$ matrices. These can be expanded in the following general way

$$
A=\sum_{n, m=1}^{N} e^{(n m)} a_{n m}
$$

where $e^{(n m)}$ are $N^{2}$ matrices defined by

$$
e_{i j}^{(n m)}=\delta_{i}^{n} \delta_{j}^{m}, \quad i . j=1, \cdots, N
$$


These special matrices satisfy the algebra

$$
e^{(n m)} e^{(p q)}=\delta_{m p} e^{(n q)}
$$

Therefore the structure constants of the algebra are given by

$$
f_{(n m)(p q)(r s)}=\delta_{m p} \delta_{n r} \delta_{q s}
$$

Recalling the definitions given in eq. (2.6), we have

$$
\left(R_{(p q)}\right)_{(n m)(r s)}=\delta_{p m} \delta_{q s} \delta_{n r}, \quad\left(L_{(p q)}\right)_{(n m)(r s)}=\delta_{p r} \delta_{q n} \delta_{m s}
$$

The matrix $C$ can be found by requiring that $\langle x C|$ is an eigenstate of $R_{i}$, that is

$$
[F(e)]^{(n m)}\left(R_{(p q)}\right)_{(n m)(r s)}=e^{(p q)}[F(e)]^{(r s)}
$$

where

$$
F(e)^{(n m)}=e^{(r s)} C_{(r s)(n m)}
$$

We get

$$
[F(e)]^{(r p)} \delta_{q s}=e^{(p q)}[F(e)]^{(r s)}
$$

By looking at the eq. (4.3), we see that this equation is satisfied by

$$
[F(e)]^{(r s)}=e^{(s r)}
$$

It follows

$$
C_{(m n)(r s)}=\delta_{m s} \delta_{n r}
$$

It is seen easily that $C$ satisfies

$$
C^{T}=C^{*}=C, \quad C^{2}=1
$$

Therefore the matrix algebra is a self-conjugated one. One easily checks that the right multiplications satisfy eq. (3.11), and therefore $C$ is an involution. More precisely, since

$$
e^{(m n)^{*}}=e^{(p q)} C_{(p q)(m n)}=e^{(n m)}
$$

the involution is nothing but the hermitian conjugation

$$
A^{*}=A^{\dagger}, \quad A \in \mathcal{A}_{N}
$$


The integration rules give

$$
\left(C^{-1}\right)_{(r p)(q s)}=\delta_{r s} \delta_{p q}=\int_{(e)} e^{(r p)} e^{(q s)}=\delta_{p q} \int_{(e)} e^{(r s)}
$$

We see that this is satisfied by

$$
\int_{(e)} e^{(r s)}=\delta_{r s}
$$

This result can be obtained also using directly eq. (2.22), noticing that the identity of the algebra is given by $I=\sum_{n} e^{(n, n)}$. Therefore

$$
\int_{(e)} e^{(r s)}=\sum_{n}\left(C^{-1}\right)_{(n n)(r s)}=\sum_{n} \delta_{n s} \delta_{n r}=\delta_{r s}
$$

and, for a generic matrix

$$
\int_{(e)} A=\sum_{m, n=1}^{N} a_{n m} \int_{(e)} e^{(n m)}=\operatorname{Tr}(A)
$$

\section{Derivations}

We will discuss now the derivations on associative algebras with identity. Recall that a derivation is a linear mapping on the algebra satisfying

$$
D(a b)=(D a) b+a(D b), \quad a, b \in \mathcal{A}
$$

We define the action of $D$ on the basis elements in terms of its representative matrix, $d$,

$$
D x_{i}=d_{i j} x_{j}
$$

If $D$ is a derivation, then

$$
S=\exp (\alpha D)
$$

is an automorphism of the algebra. In fact, it is easily proved that

$$
\exp (\alpha D)(a b)=(\exp (\alpha D) a)(\exp (\alpha D) b)
$$

On the contrary, if $S(\alpha)$ is an automorphism depending on the continuous parameter $\alpha$, then from (5.4), the following equation defines a derivation

$$
D=\lim _{\alpha \rightarrow 0} \frac{S(\alpha)-1}{\alpha}
$$


In our formalism the automorphisms play a particular role. In fact, from eq. (5.4) we get

$$
S(\alpha)\left(|x\rangle x_{i}\right)=(S(\alpha)|x\rangle)\left(S(\alpha) x_{i}\right)
$$

and therefore

$$
R_{i}(S(\alpha)|x\rangle)=S(\alpha)\left(R_{i}|x\rangle\right)=S(\alpha)\left(|x\rangle x_{i}\right)=(S(\alpha)|x\rangle)\left(S(\alpha) x_{i}\right)
$$

meaning that $S(\alpha)|x\rangle$ is an eigenvector of $R_{i}$ with eigenvalue $S(\alpha) x_{i}$. This equation shows that the basis $x_{i}^{\prime}=S(\alpha) x_{i}$ satisfies an algebra with the same structure constants as those of the basis $x_{i}$. Therefore the matrices $R_{i}$ and $L_{i}$ constructed in the two basis, and as a consequence the $C$ matrix, are identical. In other words, our formulation is invariant under automorphisms of the algebra (of course this is not true for a generic change of basis). The previous equation can be rewritten in terms of the matrix $s(\alpha)$ of the automorphism $S(\alpha)$, as

$$
R_{i}(s(\alpha)|x\rangle)=(s(\alpha)|x\rangle) s_{i j} x_{j}=s_{i j} s(\alpha) R_{j}|x\rangle
$$

or

$$
s(\alpha)^{-1} R_{i} s(\alpha)=R_{S(\alpha) x}
$$

If the algebra has an identity element, $I$, (say $x_{0}=I$ ), then

$$
D x_{0}=0
$$

and therefore

$$
D x_{0}=d_{0 i} x_{i}=0 \Longrightarrow d_{0 i}=0
$$

We will prove now some properties of the derivations. First of all, from the basic defining equation (5.1) we get

$$
\begin{aligned}
R_{i} d|x\rangle & =R_{i} D|x\rangle=D\left(R_{i}|x\rangle=D\left(|x\rangle x_{i}\right)\right. \\
& =d|x\rangle x_{i}+|x\rangle D x_{i}=d R_{i}|x\rangle+R_{D x_{i}}|x\rangle
\end{aligned}
$$

from which

$$
\left[R_{i}, d\right]=R_{D x_{i}}
$$

which is nothing but the infinitesimal version of eq. (5.9). From the integration rules (for an algebra with identity) we get immediately

$$
\int_{(x)} D x_{i}=d_{i j} \int_{(x)} x_{j}=d_{i j}\left(C^{-1}\right)_{0 j}
$$


Showing that in order that the derivation $D$ satisfies the integration by parts rule for any function, $f(x)$, on the algebra

$$
\int_{(x)} D(f(x))=0
$$

the necessary and sufficient condition is

$$
d_{i j}\left(C^{-1}\right)_{0 j}=0
$$

implying that the $d$ matrix must be singular and have $\left(C^{-1}\right)_{j 0}$ as a null eigenvector.

Next we show that, if a derivation satisfies the integration by part formula (5.15), then the matrix of related automorphism $S(\alpha)=\exp (\alpha D)$ obeys the equation

$$
C^{-1} s^{T}(\alpha) C=s^{-1}(\alpha)
$$

and it leaves invariant the measure of integration. The converse of this theorem is also true. Let us start assuming that $D$ satisfies eq. (5.15), then

$$
\begin{aligned}
0 & =\int_{(x)} D(|x\rangle\langle x| C)=\int_{(x)} d|x\rangle\left\langle x\left|C+\int_{(x)}\right| x\right\rangle\langle D x| C \\
& =d+\int_{(x)}|x\rangle\langle x| C C^{-1} d^{T} C=d+C^{-1} d^{T} C
\end{aligned}
$$

that is

$$
d+C^{-1} d^{T} C=0
$$

The previous expression can be exponentiated getting

$$
C^{-1} \exp \left(\alpha d^{T}\right) C=\exp (-\alpha d)
$$

from which the equation (5.17) follows, for $s(\alpha)=\exp (\alpha d)$. To show the invariance of the measure, let us consider the following identity

$$
1=\int_{(x)} s|x\rangle\left\langle x C\left|s^{-1}=\int_{(x)} s\right| x\right\rangle\left\langle x\left|s^{T} C=\int_{(x)}\right| S x\right\rangle\left\langle S x\left|C=\int_{(x)}\right| x^{\prime}\right\rangle\left\langle x^{\prime} C\right|
$$

where $x^{\prime}=S x$, and we have used eq. (5.17). For any automorphism of the algebra we have

$$
\int_{\left(x^{\prime}\right)}\left|x^{\prime}\right\rangle\left\langle x^{\prime} C\right|=1
$$


since the numerical values of the matrices $R_{i}$ and $L_{i}$, and consequently the $C$ matrix, are left invariant. Comparing eqs. (5.21) and (5.22) we get

$$
\int_{\left(x^{\prime}\right)}=\int_{(x)}
$$

On the contrary, if the measure is invariant under an automorphism of the algebra, the chain of equalities

$$
1=\int_{\left(x^{\prime}\right)}\left|x^{\prime}\right\rangle\left\langle x^{\prime} C\left|=\int_{(x)}\right| x^{\prime}\right\rangle\left\langle x^{\prime} C\left|=\int_{(x)} s\right| x\right\rangle\langle x| C\left(C^{-1} s^{T} C\right)=s\left(C^{-1} s^{T} C\right)
$$

implies eq. (5.17), together with its infinitesimal version eq. (5.19). From this (see the derivation in (5.18)), we get

$$
0=\int_{(x)} D\left(x_{i} x_{j} C_{j k}\right)
$$

and by taking $x_{i}=I$,

$$
\int_{(x)} D x_{j}=0
$$

for any basis element of the algebra. Therefore we have proven the following theorem:

If a derivation $D$ satisfies the integration by part rule, eq. (5.15), the integration is invariant under the related automorphism $\exp (\alpha D)$. On the contrary, if the integration is invariant under a continuous automorphism, $\exp (\alpha D)$, the related derivation, $D$, satisfies (5.15).

This theorem generalizes the classical result about the Riemann integral relating the invariance under translations of the measure and the integration by parts formula.

Next we will show that, always in the case of an associative self-conjugated algebra, $\mathcal{A}$, with identity, there exists a set of automorphisms such that the measure of integration is invariant. These are the so called inner derivations, that is derivations such that

$$
D \in \mathcal{L}(\mathcal{A})
$$


where $\mathcal{L}(\mathcal{A})$ is the Lie multiplication algebra associated to $\mathcal{A}$. To define $\mathcal{L}(\mathcal{A})$ one starts with the linear space of left and right multiplications and defines

$$
\mathcal{M}_{1}=\mathcal{M}_{R}+\mathcal{M}_{L^{T}}
$$

that is the space generated by the vectors

$$
R_{a}+L_{b}^{T}, \quad a, b \in \mathcal{A}
$$

Then

$$
\mathcal{L}(\mathcal{A})=\sum_{i=1}^{\infty} \mathcal{M}_{i}
$$

where the spaces $\mathcal{M}_{i}$ are defined by induction

$$
\mathcal{M}_{i+1}=\left[\mathcal{M}_{1}, \mathcal{M}_{i}\right]
$$

Therefore $\mathcal{L}(\mathcal{A})$ is defined in terms of all the multiple commutators of the elements given in (5.29).

It is not difficult to prove that for a Lie algebra, $\mathcal{L}(\mathcal{A})$ coincides with the adjoint representation [9]. We will prove now an analogous result for associative algebras with identity. That is that $\mathcal{L}(\mathcal{A})$ coincides with the adjoint representation of the Lie algebra associated to $\mathcal{A}$ (the Lie algebra generated by $[a, b]=a b-b a$, for $a, b \in \mathcal{A})$. The proof can be found, for example, in ref. [9], but for completeness we will repeat it here. From the associativity conditions (2.8), and (2.13) one gets

$$
\left[R_{a}+L_{b}^{T}, R_{c}+L_{d}^{T}\right] \in \mathcal{M}_{1}, \quad a, b, c, d \in \mathcal{A}
$$

or

$$
\left[\mathcal{M}_{1}, \mathcal{M}_{1}\right] \subset \mathcal{M}_{1}
$$

showing that

$$
\mathcal{L}(\mathcal{A})=\mathcal{M}_{1}=\mathcal{M}_{R}+\mathcal{M}_{L^{T}}
$$

Therefore the matrix associated to an inner derivation of an associative algebra must be of the form

$$
d=R_{a}+L_{b}^{T}
$$

We have now to require that this indeed a derivation, that is that eq. (5.13) holds. We start evaluating

$$
\left[R_{c}, d\right]=\left[R_{c}, R_{a}+L_{b}^{T}\right]=R_{[c, a]}
$$


where we have used the fact that the right multiplications form a representation of the algebra and that right and left multiplications commute. Then comparing with

$$
R_{D c}=R_{c a+c b}
$$

we see that the two agree for $b=-a$. Then we get

$$
D x_{i}=x_{i} a-a x_{i}=-\left[a, x_{i}\right]=-(\operatorname{adj} a)_{i j} x_{j}
$$

This shows indeed that the inner derivations span the adjoint representation of the Lie algebra associated to $\mathcal{A}$.

We can now proof the following theorem:

For an associative self-conjugated algebra with identity, the measure of integration is invariant under the automorphisms generated by the inner derivations, or, equivalently, the inner derivations satisfy the rule of integration by parts.

In fact, this follows because the inner derivations satisfy eq. (5.19)

$$
C^{-1} d^{T} C=C^{-1}\left(R_{a}^{T}-L_{a}\right) C=\left(C^{T} R_{a} C^{T^{-1}}\right)^{T}-R_{a}=L_{a}^{T}-R_{a}=-d
$$

As an example let us consider the algebra of the matrices studied in the previous Section. In this case the inner derivations are simply given by

$$
D_{B} A=[A, B]
$$

Therefore

$$
\int_{(e)} D_{B} A=\int_{(e)}[A, B]=0
$$

and we see that the integration by parts formula corresponds to the cyclic property of the trace.

\section{Paragrassmann algebras as subalgebras of an algebra of matrices}

Since an associative algebra can be represented in terms of matrices, and having shown that, in this case, the integration is simply given by the trace, 
one can ask if it is possible to use this result in order to get the integration over a subalgebra of $\mathcal{A}_{N}$. The idea is simply that one should integrate with the trace formula, but using a weight which selects the particular subalgebra one is interested to. We will illustrate this procedure for a paragrassmann algebra of order $p$, that is an algebra generated by an element $\theta$, such that

$$
\theta^{p+1}=0
$$

For $p=1$ we get a Grassmann algebra. Any element of the algebra is given by a power of $\theta$

$$
x_{k}=\theta^{k}, \quad k=0,1, \cdots, p
$$

Being the algebra an associative one, the elements $\theta^{k}$ can be represented in terms of the right multiplication matrices, $R_{k}$. These are $(p+1) \times(p+1)$ matrices given by (see 四)

$$
\left(R_{i}\right)_{j k}=\delta_{i+j, k}
$$

Defining

$$
X_{\theta} \equiv R_{1}
$$

we can write, in terms of the matrices defined in eq. (4.2)

$$
X_{\theta}=\sum_{i=1}^{p} e^{(i, i+1)}
$$

and

$$
X_{\theta}^{k}=\sum_{i=1}^{p+1-k} e^{(i, i+k)}
$$

Therefore, the most general function on the paragrassmann algebra (as a subalgebra of the matrices $(p+1) \times(p+1))$ is given by

$$
f\left(X_{\theta}\right)=\sum_{i=1}^{p+1} a_{i} X_{\theta}^{p+1-i}=\sum_{i=1}^{p+1} a_{i} \sum_{j=1}^{i} e^{(j, p+1+j-i)}
$$

As we said, the idea is to look for a matrix $P$ such that it projects out of the algebra, $\mathcal{A}_{p+1}$ of the $(p+1) \times(p+1)$ matrices, the paragrassmann subalgebra. To define such an operator, let us consider a generic matrix $B \in \mathcal{A}_{p+1}$. We can always decompose it as (see later)

$$
B=f\left(X_{\theta}\right)+\tilde{B}
$$


The operator $P$ should satisfy

$$
B P=f\left(X_{\theta}\right) P
$$

or

$$
\tilde{B} P=0
$$

Then, we can define the integration over the paragrassmann algebra in terms of the integration over the isomorphic subalgebra of $\mathcal{A}_{p+1}$ through the equation

$$
\int_{(\theta)} f(\theta)=\int_{(e)} f\left(X_{\theta}\right) P=\operatorname{Tr}\left[f\left(X_{\theta}\right) P\right]
$$

In order to define the decomposition (6.8) and the operator $P$, let us consider the most general $(p+1) \times(p+1)$ matrix. We can write

$$
B=\sum_{i, j=1}^{p+1} b_{i j} e^{(i j)}=\sum_{i=1}^{p+1} \sum_{j=1}^{p} b_{i j} e^{(i j)}+\sum_{i=1}^{p+1} b_{i, p+1} e^{(i, p+1)}
$$

By adding and subtracting

$$
\sum_{i=2}^{p+1} b_{i, p+1} \sum_{j=1}^{i-1} e^{(j, p+1+j-i)}
$$

we get the decomposition (6.8) with

$$
f\left(X_{\theta}\right)=\sum_{i=1}^{p+1} b_{i, p+1} X_{\theta}^{p+1-i}
$$

and

$$
\tilde{B}=\sum_{i=1}^{p+1} \sum_{j=1}^{p} b_{i j} e^{(i j)}-\sum_{i=2}^{p+1} b_{i, p+1} \sum_{j=1}^{i-1} e^{(j, p+1+j-i)}
$$

Let us notice that for any integer $k, 1 \leq k \leq p+1$, we have

$$
\tilde{B} e^{(p+1, k)}=0
$$

or

$$
B e^{(p+1, k)}=f\left(X_{\theta}\right) e^{(p+1, k)}
$$

But using the identity

$$
e^{(p+1, k)}=e^{(p+1,1)} X_{\theta}^{k-1}
$$


we get

$$
\operatorname{Tr}\left[B e^{(p+1, k)}\right]=\operatorname{Tr}\left[X_{\theta}^{k-1} f\left(X_{\theta}\right) e^{(p+1,1)}\right] \equiv \operatorname{Tr}\left[g\left(X_{\theta}\right) e^{(p+1,1)}\right]
$$

where $g\left(x_{\theta}\right)=X^{k-1} f\left(X_{\theta}\right)$. This shows that we can always define the integration trough the operator $P=e^{(p+1,1)}$. Then, the integration over the subalgebra is given by

$$
\int_{\theta} f(\theta)=\int_{(e)} f\left(X_{\theta}\right) e^{(p+1,1)}=\operatorname{Tr}\left[f\left(X_{\theta}\right) e^{(p+1,1)}\right]
$$

It follows from eq. (6.14), and eq. (4.15)

$$
\int_{(\theta)} f(\theta)=\operatorname{Tr}\left[\sum_{i=1}^{p+1} b_{i, p+1} e^{(i, 1)}\right]=b_{1, p+1}
$$

Meaning that

$$
\int_{(\theta)} f(\theta)=\int_{(\theta)}\left[b_{1, p+1} \theta^{p}+b_{2, p+1} \theta^{p-1}+\cdots+b_{p+1, p+1}\right]=b_{1, p+1}
$$

or

$$
\int_{(\theta)} \theta^{k}=\delta_{k p}
$$

which coincides with the direct way of defining the integral over a paragrassmann algebra (see [4]). Of course, by choosing $P=e^{(p+1, k)}$ with $k \neq 1$ would lead to an integral, which can be expressed in terms of the one in eq. (6.23), as

$$
\int_{(\theta)} \theta^{k-1} f(\theta)
$$

In the particular case of a Grassmann algebra we have

$$
X_{\theta}=e^{(1,2)}=\left(\begin{array}{ll}
0 & 1 \\
0 & 0
\end{array}\right)=\sigma_{+}, \quad P=e^{(2,1)}=\left(\begin{array}{ll}
0 & 0 \\
1 & 0
\end{array}\right)=\sigma_{-}
$$

The decomposition in eq. (6.8), for a $2 \times 2$ matrix

$$
B=a+b \sigma_{3}+c \sigma_{+}+d \sigma_{-}
$$

is given by

$$
\tilde{B}=b\left(1+\sigma_{3}\right)+d \sigma_{-}, \quad f\left(X_{\theta}\right)=f\left(\sigma_{+}\right)=a-b+c \sigma_{+}
$$


and the integration is

$$
\int_{(\theta)} f(\theta)=\operatorname{Tr}\left[f\left(\sigma_{+}\right) \sigma_{-}\right]
$$

from which

$$
\int_{(\theta)} 1=\operatorname{Tr}\left[\sigma_{-}\right]=0, \quad \int_{(\theta)} \theta=\operatorname{Tr}\left[\sigma_{+} \sigma_{-}\right]=1
$$

We notice that the matrices $\tilde{B}$ and $f\left(X_{\theta}\right)$ appearing in the decomposition (6.8) can be written more explicitly as

$$
\tilde{B}=\left(\begin{array}{ccccc}
\tilde{b}_{1,1} & \tilde{b}_{1,2} & \cdots & \tilde{b}_{1, p} & 0 \\
\cdot & \cdot & \cdot & \cdot & \cdot \\
\cdot & \cdot & \cdot & \cdot & \cdot \\
\tilde{b}_{p, 1} & \tilde{b}_{p, 2} & \cdots & \tilde{b}_{p, p} & 0 \\
\tilde{b}_{p+1,1} & \tilde{b}_{p+1,2} & \cdots & \tilde{b}_{p+1, p} & 0
\end{array}\right)
$$

and

$$
f\left(X_{\theta}\right)=\left(\begin{array}{cccccc}
a_{p+1} & a_{p} & a_{p-1} & \cdots & a_{2} & a_{1} \\
0 & a_{p+1} & a_{p} & \cdots & a_{3} & a_{2} \\
0 & 0 & a_{p+1} & \cdots & a_{4} & a_{3} \\
\cdot & \cdot & \cdot & \cdot & \cdot & \cdot \\
\cdot & \cdot & \cdot & \cdot & \cdot & \cdot \\
0 & 0 & 0 & \cdots & a_{p} & a_{p-1} \\
0 & 0 & 0 & \cdots & a_{p+1} & a_{p} \\
0 & 0 & 0 & \cdots & 0 & a_{p+1}
\end{array}\right)
$$

The $p \times(p+1)$ parameters appearing in $\tilde{B}$ and the $p+1$ parameters in $f\left(X_{\theta}\right)$ can be easily expressed in terms of the $(p+1) \times(p+1)$ parameters defining the matrix $B$.

\section{Conclusions}

In this paper we have studied some general properties of the integration over self-conjugated associative algebra with identity. We have proven a theorem showing that continuous automorphisms, leaving invariant the measure of integration, give rise to derivations satisfying the integration by part rule 
(that is the vanishing of the integral of a derivative). The relevance of this result is that in quantum mechanics the Schwinger's principle follows trivially from the previous theorem, therefore it opens the avenue to extensions to more general theories.

The other important problem we have considered is the following: given the integral over an algebra, is there a natural way to induce the integral over a subalgebra? To face this problem we have followed the way suggested by the standard integration over manifolds, that is to project out (via the characteristic function) the submanifold in the measure. We have illustrated this procedure for a paragrassmann algebra of order $p$, thought as a subalgebra of an algebra of $(p+1) \times(p+1)$ matrices, showing that it is possible define a projector selecting the paragrassmann component out of a given matrix. Since in the text we have shown that the integral for an algebra of matrices coincides with the trace, we arrive to evaluate the integral over a paragrassmann algebra via a trace of ordinary matrices. In particular the integration over the one-dimensional Grassmann algebra can be expressed as a trace of $2 \times 2$ matrices. This result might be of some interest for fermionic theories on the lattice.

\section{Acknowledgements}

The author would like Prof. J. P. Eckmann, Director of the Department of Theoretical Physics of the University of Geneva, for the very kind hospitality. 


\section{References}

[1] Y.A. Golfand and E.S. Likhtman, JETP Lett. 13 (1071)313; D.V. Volkov and V.P. Akulov, Pis'ma Zh. Eksp. Teor. Fiz. 16 (1972) 621; ibidem Phys. Lett. B46 (1973) 109; J. Wess and B. Zumino, Nucl. Phys. B70 (1974) 139.

[2] R. Casalbuoni, Il Nuovo Cimento, 33A (1976) 389.

[3] T. Banks, W. Fischler, S.H. Shenker and L. Susskind, Phys. Rev. D55 (1997) 5112, hep-th/9610043.

[4] R.Casalbuoni, Int. J. Mod. Phys. A12 (1997) 5803, physics/9702019.

[5] A. Connes, Noncommutative geometry, Academic Press (1994).

[6] V.G. Drinfeld, Quantum Groups, in Proceedings of the International Congress of Mathematicians, Berkeley 1986, pp. 798-820, AMS, Providence, RI.

[7] R. Casalbuoni, hep-th/9801170.

[8] R. Casalbuoni, math-phys/9804004.

[9] R.D. Schafer, An introduction to nonassociative algebras, Academic Press (1966). 